\title{
Noncoding RNAs involved in the STAT3 pathway in glioma
}

\author{
Zheng Bian ${ }^{\dagger}$, Wei $\mathrm{Ji}^{\dagger}$, Bin $\mathrm{Xu}^{\dagger}$, Zhengyuan Huo, Hui Huang, Jin Huang, Jiantong Jiao, Junfei Shao* and \\ Xiaolu Zhang*(1)
}

\begin{abstract}
Glioma is the most common malignant primary brain tumour in adults. Despite improvements in neurosurgery and radiotherapy, the prognosis of glioma patients remains poor. One of the main limitations is that there are no proper clinical therapeutic targets for glioma. Therefore, it is crucial to find one or more effective targets. Signal transducer and activator of transcription 3 (STAT3) is a member of the STAT family of genes. Abnormal expression of STAT3 is involved in the process of cell proliferation, migration, invasion, immunosuppression, angiogenesis, dryness maintenance, and resistance to radiotherapy and chemotherapy in glioma. Therefore, STAT3 has been considered an ideal therapeutic target in glioma. Noncoding RNAs (ncRNAs) are a group of genes with limited or no protein-coding capacity that can regulate gene expression at the epigenetic, transcriptional and posttranscriptional level. In this review, we summarized the ncRNAs that are correlated with the ectopic expression of STAT3 in glioma.
\end{abstract}

Keywords: glioma, miRNA, IncRNAs, circRNAs, STAT3

\section{Introduction}

Glioma is the most common primary intracranial malignant tumour. Despite the great progress that has been made in neurosurgical technology, radiotherapy and chemotherapy, the prognosis of glioma patients is still poor [1]. Particularly in glioblastoma (GBM), the 5-year survival rate of patients is still less than $5 \%$, and the median survival time is less than 1 year [2]. One of the main reasons for the poor prognosis of glioma is that there are no specific and effective therapeutic targets in clinical practice. Therefore, elucidating the molecular mechanism underlying tumorigenesis and identifying novel therapeutic targets are essential for the treatment of glioma.

Noncoding RNAs (ncRNAs) are a group of genes with limited or no protein-coding capacity [3]. Based on their length, ncRNAs are divided into long noncoding RNAs

\footnotetext{
*Correspondence: wxrmyysjf@163.com; wxrmyyzxl@163.com

'Zheng Bian, Wei Ji and Bin Xu contributed equally to this work Department of Neurosurgery, Wuxi People's Hospital of Nanjing Medical University, Wuxi, People's Republic of China
}

(>200 nt) and small noncoding RNAs. Small noncoding RNAs can be divided into small nuclear RNAs (snRNAs), small nucleolar RNAs (snoRNAs), microRNAs (miRNAs), and piRNAs. Noncoding RNAs account for $60 \%$ of human transcripts, and they were previously thought to be genomic "dark matter" [4]. However, an increasing number of studies have found that noncoding RNAs play important roles in the physiological and pathological processes of various human diseases, including tumours $[5,6]$. The role of noncoding RNAs in glioma has also been widely investigated. Studies have found that noncoding RNAs are involved in a series of processes, such as the occurrence, development, and treatment resistance of gliomas, and have become a potential prognostic, diagnostic indicator, and treatment target $[7,8]$.

Signal transducer and activator of transcription 3 (STAT3) is a member of the signal transducer and activator of transcription (STAT) protein family, which can be activated by a variety of cytokines or growth factors [9]. STAT protein family members contain an amino end domain, coiled-coil domain, DNA-binding original author(s) and the source, provide a link to the Creative Commons licence, and indicate if changes were made. The images or other third party material in this article are included in the article's Creative Commons licence, unless indicated otherwise in a credit line to the material. If material is not included in the article's Creative Commons licence and your intended use is not permitted by statutory regulation or exceeds the permitted use, you will need to obtain permission directly from the copyright holder. To view a copy of this licence, visit http://creativecommons.org/licenses/by/4.0/. The Creative Commons Public Domain Dedication waiver (http://creativecommons.org/publicdomain/zero/1.0/) applies to the data made available in this article, unless otherwise stated in a credit line to the data. 
domains (DBD), Src homology 2 domain (SH2) domain, tyrosine activation domain and transcription activation domain (TAD). The activation of STAT3 can be induced by epidermal growth factor receptor (EGFR), JAK2, and other tyrosine kinases activated by epidermal growth factor (EGF), leukaemia inhibitory factor (LIF), and other cytokines [10]. Upon activation, STAT3 can form homodimers or heterodimers (STAT1/3) and enter the nucleus. Then, homodimers or heterodimers interact with the corresponding target gene promoter to activate gene transcription and translation. Janus kinase (JAK), acting as a transcription factor, plays an important role in the activation of STAT3. The classic signalling cascade of the JAK/STAT pathway involves the dimerization of receptor molecules caused by the cytokine binding to its receptor leading to the coupling of JAK receptors, which are brought into close proximity and then activated by interactive tyrosine phosphorylation. Following JAK activation, JAKs catalyse the tyrosine phosphorylation of the receptor itself and form corresponding STAT docking sites. Upon the activation of JAKs, STAT3 binds to receptors through the SH2 domain and is phosphorylated [11].

The expression and activation of STAT3 are precisely regulated, including its proteasomal degradation and the activation-inactivation mechanism that regulates the transient activation of STAT3 [12]. It is clear that the suppressor of cytokine signalling (SOCS) protein family and the protein inhibitor of activated STAT (PIAS) protein family are important negative regulators of the STAT signalling pathway. Among them, SOCS can inhibit STAT signal transduction by binding and inhibiting JAKs [13]. PIAS only binds to activated STAT dimers. By recruiting acetylase, it promotes the SUMO (small ubiquitin-like modifier) modification of STAT and induces dimerization and dissociation to inhibit the DNA binding and transactivation ability of STAT3 [14]. In normal cells, the activation of STAT3 is transient, but in gliomas, due to the lack of upstream regulatory signals and negative regulatory mechanisms, STAT3 is constitutively highly expressed and activated. The high expression and activation of STAT3 play an important role in gliomas. For example, in vivo and in vitro experiments have shown that c-myc, CCND1, Bcl-2, BCL-XL, Survivin, etc., are the direct target genes of STAT3 in the regulation of glioma cell proliferation $[15,16]$. STAT3 regulates the migration and invasion of glioma cells by targeting MMP2, MMP9, SNAIL, etc. [17]. STAT3 regulates angiogenesis by targeting VEGF [18]. In addition, STAT3 regulates the activity and proliferation of $\mathrm{T}$ cells and the function of dendritic cells to participate in tumour immunity $[19,20]$. STAT3 also plays a significant role in the resistance to radiotherapy and chemotherapy of glioma [21, 22].
In glioma, ncRNAs can play an important role in upstream signals and mechanisms to regulate the expression and activation of STAT3. In addition, STAT3, acting as a transcription factor, can directly target and then regulate the expression of ncRNAs. To further understand the occurrence of glioma and provide new ideas for the development of STAT3-targeted tumour therapy, this article mainly summarizes the ncRNAs involved in the STAT3-mediated signalling pathway in glioma.

\section{MiRNAs involved in the STAT3 signalling pathway}

MiRNAs are endogenous, noncoding small RNAs with an approximate length of 20-22 nucleotides that mainly participate in regulating gene transcription, translation, or epigenetic processes. They are widely found in eukaryotes, with a high degree of conservation, cell and tissue specificity and timing [23]. The production of miRNAs is a multistep process and involves the canonical biogenesis pathway. In the nucleus, the miRNA gene is transcribed into a primary miRNA transcript (pri-miRNA) that is 300-1000 base pairs (bp) in length by RNA polymerase II [24]. Mediated by the RNase III Drosha, pri-miRNAs are cleaved to pre-miRNAs to a length of approximately $70 \mathrm{bp}$ and contain a stem-loop structure [25]. Then, premiRNAs are transported from the nucleus to the cytoplasm by the Ran-GTP-dependent transporter exportin 5 (XPO5) [26]. In the cytoplasm, Dicer, a double-stranded RNA-specific endonuclease, and TAR RNA-binding protein (TRBP) recognize pre-miRNAs. Pre-miRNAs are further processed by Dicer to generate mature doublestranded miRNAs with a length of 20-22 nucleotides. To regulate gene expression, mature double-stranded miRNAs unwind; then, one of the strands is degraded, and the other is integrated into the RNA-induced silencing complex (RISC) and guides it to the target mRNA. Finally, the complex binds to its target mRNA, inhibiting gene expression [27, 28].

MiRNAs play important regulatory roles in eukaryotes by binding to corresponding mRNA transcripts and then leading to their degradation and/or translation inhibition at the mRNA level [29]. Studies have shown that miRNAs can function not only as a single entity but also in the form of gene clusters. A miRNA gene cluster refers to a gene group composed of two or more miRNAs that are closely adjacent to each other on a chromosome. The miRNAs arranged in clusters may have a homologous relationship, or they may not show any homology. Meanwhile, the expression patterns of miRNAs arranged in clusters are highly consistent and have coordinated regulatory effects [30]. The most common mechanism underlying gene expression regulation by a mature miRNA is through its interaction with the $3^{\prime}$-untranslated region (UTR) of the target mRNA or the complementary 
sequence of the coding region leading to mRNA degradation or translation inhibition $[31,32]$. In addition, studies have shown that miRNAs could also interact with the $5^{\prime}$-UTR, coding sequences and the promoter regions of their target genes. MiRNA binding to the $5^{\prime}$-UTR of the target gene will promote its transcription, which is the opposite of what occurs when a miRNA binds to the 3'-UTR [33]. In addition, in recipient cells, miRNAs can be secreted and regulate target gene expression via the exosomal pathway [34] (Fig. 1).

An increasing number of studies have shown that miRNAs are involved in the occurrence and development of gliomas by acting alone or in the form of gene clusters. These miRNAs may regulate the expression of nearly one-third of the genes in the genome. Different miRNAs are involved in distinct pathophysiological processes, including the proliferation, migration, invasion, apoptosis, radiotherapy, and chemotherapy resistance of glioma cells. The occurrence of glioma is closely related to the increase in the expression of tumour-promoting miRNAs and the decrease in the expression of tumour-suppressing
miRNAs [35]. In addition, due to the stability of miRNA structure, its role as a diagnostic and prognostic indicator of glioma is receiving increasing attention [36]. The use of miRNA mimics and anti-miRNA oligonucleotides to treat tumours in animals has achieved clear results. Therefore, miRNAs are also considered potential targets for tumour therapy. The miRNAs related to the STAT3 signalling pathway in gliomas can be divided into the following types as shown in Table 1.

\section{MiRNAs directly targeting STAT3}

In GBM, on the one hand, miRNAs can directly target STAT3 to regulate the expression of related proteins in the STAT3 pathway, thereby affecting tumour cell function [37]. In terms of glioma cell proliferation, the B-cell lymphoma/leukaemia-2 gene (bcl-2) plays an important role in tumour cell anti-apoptosis, promotes tumour cell proliferation, and inhibits tumour cell apoptosis [38]. LC3 is a significant marker of autophagy, while P62 acts as a substrate of ubiquitination [39]. MiRNA clusters let7a-1 let-7d (including let-7d, let-7f-1 and let-7a-1) can

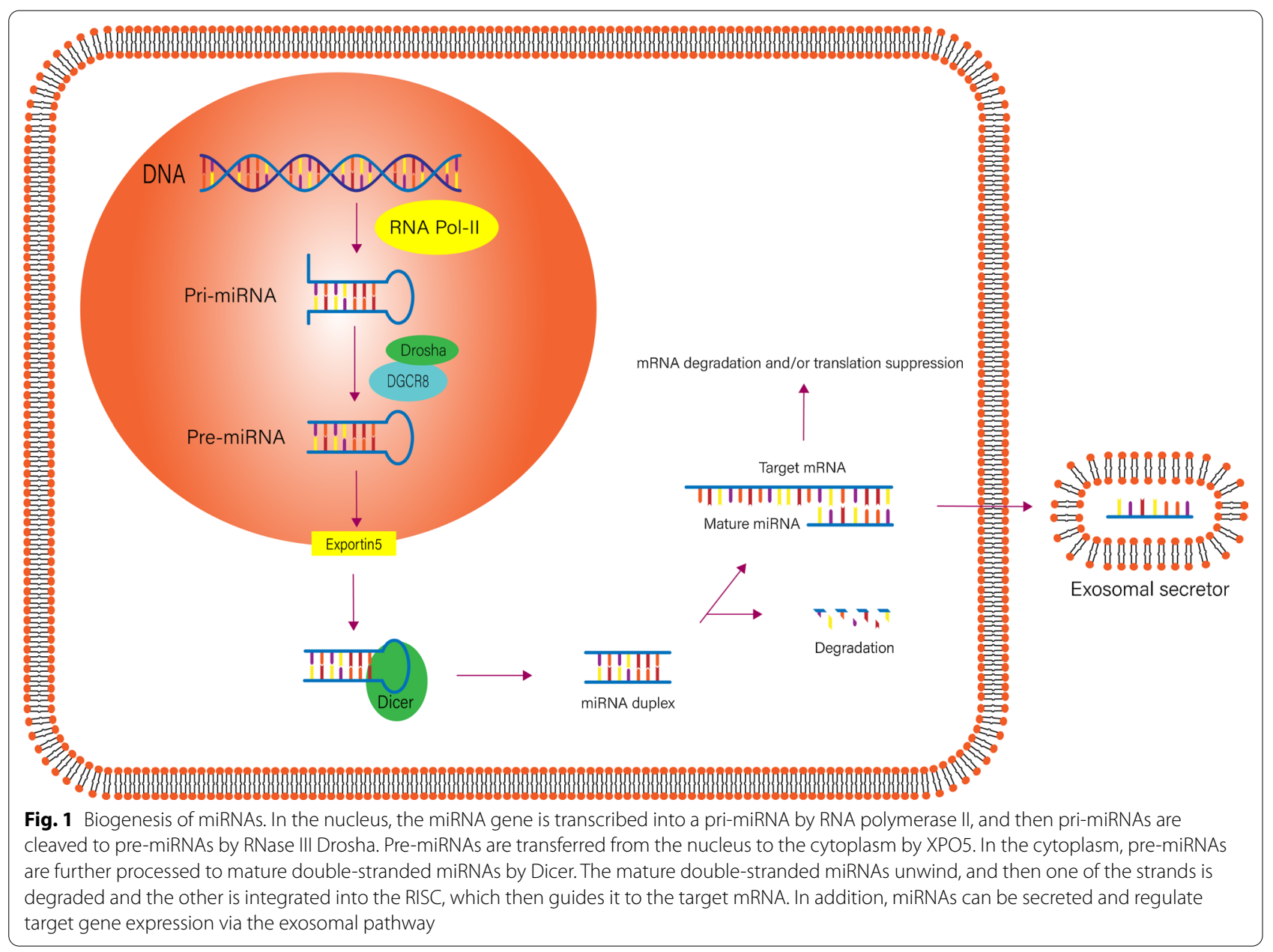


Table 1. MiRNAs involved in STAT3 signaling pathway

\begin{tabular}{|c|c|c|c|c|c|}
\hline MicroRNA & $\begin{array}{l}\text { Up-I } \\
\text { down- } \\
\text { regulation }\end{array}$ & Role in glioma & Biological functions & Target gene & Refs. \\
\hline miR cluster MC-let-7a-1 let-7d & Down & Tumor suppressor & $\begin{array}{l}\text { Inhibit proliferation, promote apoptosis, and cell } \\
\text { autophagy }\end{array}$ & STAT3 & [40] \\
\hline miR-506 & Down & Tumor suppressor & Inhibit proliferation, migration, and invasion & STAT3 & [42] \\
\hline miR-181d & Down & Tumor suppressor & Inhibit migration and invasion & STAT3/STAT5A & [43] \\
\hline miR-29b & Down & Tumor suppressor & Inhibit chemoresistance & STAT3 & {$[45]$} \\
\hline miR-519a & Down & Tumor suppressor & $\begin{array}{l}\text { Inhibit proliferation, migration, invasion, and } \\
\text { chemoresistance }\end{array}$ & STAT3 & {$[46,47]$} \\
\hline miR-124 & Down & Tumor suppressor & $\begin{array}{l}\text { Inhibit proliferation, promote apoptosis, and } \\
\text { immune therapeutics }\end{array}$ & STAT3/SAMD4 & {$[48,56]$} \\
\hline miR-221/222 cluster & Up & Oncogene & $\begin{array}{l}\text { Promote migration, invasion, proliferation, and } \\
\text { angiogenesis }\end{array}$ & sOCS3 & [50] \\
\hline miR-30 & Up & Oncogene & Promote tumorigenesis & sOCS3 & [51] \\
\hline miR-125b & Up & Oncogene & Promote chemoresistance & PIAS3 & [53] \\
\hline miR-133a & Down & Tumor suppressor & $\begin{array}{l}\text { Inhibit proliferation, migration, and invasion, } \\
\text { promote apoptosis }\end{array}$ & CTGF & [55] \\
\hline miR-148a & Up & Oncogene & Promote migration, invasion & CADM1 & [58] \\
\hline miR-876-3p & Down & Tumor suppressor & Inhibit proliferation, migration, and invasion & KIF20A & {$[60]$} \\
\hline miR-26a & Up & Oncogene & $\begin{array}{l}\text { Promote chemoresistance, migration, invasion, } \\
\text { proliferation }\end{array}$ & AP-2a & [62] \\
\hline miR-6743-5p & Up & Oncogene & Promote proliferation, inhibit apoptosis & GRIM-19 & [63] \\
\hline miR-1246 & Up & Oncogene & Promote proliferation, migration, and invasion & TERF2IP & {$[65]$} \\
\hline miR-125b/miR-20b & Up & Oncogene & Promote proliferation & FZD6/ALDH1A3 & {$[67]$} \\
\hline $\operatorname{miR}-21$ & Up & Oncogene & Promote proliferation and invasion & RECK & {$[68,69,70,71]$} \\
\hline miR-182-5p & Up & Oncogene & Promote proliferation, migration, and invasion & $\mathrm{PCDH} 8$ & [72] \\
\hline miR-30b-3p & Up & Oncogene & $\begin{array}{l}\text { Promote proliferation, chemoresistance, and } \\
\text { inhibit apoptosis }\end{array}$ & $\mathrm{RHOB}$ & [73] \\
\hline miR-218 & Down & Tumor suppressor & Inhibits proliferation & PDGFRa & [74] \\
\hline miR-34a & Down & Tumor suppressor & Inhibit migration and invasion & - & [75] \\
\hline miR-184 & Up & Oncogene & Promote proliferation and invasion & - & [76] \\
\hline miR-203 & Down & Tumor suppressor & Radiosensitivity & - & [77] \\
\hline miR-31 & Down & Tumor suppressor & Promote apoptosis & - & [78] \\
\hline miR-27b & Up & Oncogene & Promote invasion, proliferation, inhibit apoptosis & - & [79] \\
\hline miR-302/367 & Down & Tumor suppressor & Inhibit proliferation, migration & - & {$[80]$} \\
\hline
\end{tabular}

directly target and inhibit STAT3 to promote apoptosis and autophagy of glioma cells. A study confirmed that STAT3 was the direct target gene of these three let-7 miRNA dual luciferase reporters. Then, cell fluorescence staining and experiments in mouse models confirmed that miRNA clusters of let-7a-1 let-7d acted as tumour suppressors in glioma [40]. In terms of migration and invasion, the growth and metastasis of GBM depend on the expression of matrix metalloproteinase 2 (MMP2). Overexpression of MMP2 promotes the metastasis and spread of various tumours and is the most important molecule that directly promotes tumour metastasis [41]. MiR-506 directly targets STAT3 to exert a tumour suppressor effect. Functional experiments have shown that overexpression of miR-506 could inhibit the migration and invasion of glioma cells and reduce the protein expression levels of MMP2, cyclin D1 and Bcl-2 [42]. Interestingly, by using Schrodinger PyMOL 2.3 molecular docking and visualization software, it has been demonstrated that miR-181d interacts with and binds directly to STAT3 or STAT5A. Upregulating miR-181d inhibits GBM cell migration, invasion, and tumour growth in GBM mouse models by suppressing STAT3 or STAT5A expression [43].

On the other hand, miRNAs can directly target STAT3 and function as therapeutic tools for GBM treatment. In terms of chemotherapy resistance, temozolomide (TMZ) is a standard chemotherapy drug for GBM multiforme. 
However, the occurrence of TMZ resistance is increasing rapidly in clinical treatment. In recent years, miRNAs have been confirmed to act as important regulators in the process of drug resistance [44]. Overexpression of miR$29 \mathrm{~b}$ can enhance the sensitivity of TMZ-resistant glioma cells to TMZ by inhibiting the expression of STAT3 [45]. MiR-519a functions as a tumour suppressor by directly targeting STAT3 in GBM. In vitro studies have shown that miR-519a can increase the sensitivity of glioma cells to TMZ by promoting autophagy, while in vivo experiments in nude mice have also proven that miR-519a enhances the therapeutic effect of TMZ on glioma cells. In addition, the decreased expression of miR-519a is related to shorter tumour-free survival and overall survival rates. In terms of immune therapeutics, STAT3 signalling has been shown to be a key regulator of the microglia/macrophage-mediated immune response [46, 47]. MiR-124 can directly target STAT3 in glioma cells to inhibit their proliferation and promote cell apoptosis. Studies have found that miR-124 plays a therapeutic role in genetically engineered mouse models expressing STAT3 and strengthens the immune response mediated by $\mathrm{T}$ cells [48].

\section{MiRNAs regulate the expression of genes upstream of STAT3}

It has been reported that p-STAT3 is key for the function of STAT3. P-STAT3 forms homodimers or heterodimers (STAT1/STAT3) through mutual phosphotyrosine-SH2 domain interactions, enters the nucleus, and regulates the transcription of target genes. Regarding the negative regulation of STAT3 activation, the SOCS and PIAS protein families play important roles. Suppressor of cytokine signalling 3 (SOCS3) belongs to the SOCS family and harbours an $\mathrm{SH} 2$ domain, which can bind to the active region of JAKs through the $\mathrm{SH} 2$ domain, preventing it from phosphorylating STAT3 [49]. RT-qPCR found that miR-221 and miR-222 are highly expressed in glioma cell lines. Both in vivo and in vitro experiments have shown that inhibition of the miR-221/222 cluster can inhibit cell migration, invasion, proliferation, and angiogenesis. Therefore, miR-221 and miR-222 are considered to act as oncogenes in gliomas. In terms of the mechanism, the miR-221/222 cluster directly targets SOCS3 and inhibits its expression, resulting in an increase in the protein level of p-STAT3 [50]. In addition, miR-30 was identified as a potential oncogene in GBM. MiR-30 can also increase the protein expression of $\mathrm{p}$-STAT3 to promote tumorigenesis by targeting SOCS3 [51]. Protein inhibitor of activated STAT3 (PIAS3) binds to an activated homodimer or heterodimer (STAT1/STAT3) and then blocks STAT3 DNA-binding activity [52]. In glioblastoma stem cells (GSCs), the expression level of miR-125b is high. A
miR-125b inhibitor enhanced the invasion-prevention activity of temozolomide in GSCs by targeting PIAS3, which then reduced STAT3 transcriptional activity and subsequently decreased the expression of matrix metalloproteinases MMP2 and MMP9 [53].

In addition to SOCS3 and PIAS3, miRNAs can regulate growth factor/cytokine receptors, which are common ways to activate STAT3. Connective tissue growth factor (CTGF) is related to the occurrence and development of tumours; participates in cell proliferation, development, adhesion, migration, and angiogenesis; and predicts prognosis [54]. MiRNA-133a, which is expressed at low levels in human glioma tissue and glioma cells, targets CTGF and then regulates the JAK/STAT signalling pathway by acting as a tumour suppressor gene [55]. Transforming growth factor- $\beta$ (TGF- $\beta$ ), which functions as an oncogene in glioma, can activate the Jak/Stat 3 pathway in a Smad-dependent manner. In addition to directly targeting STAT3, miR-124 regulates the protein levels of STAT3 and p-STAT3 to inhibit cell proliferation by targeting Smad4 [56]. Additionally, it has been reported that cell adhesion molecule 1 (CADM1) can interact with HER2 and Itga6b4 to reduce STAT3 pathway activity [57]. MiR-148a targets CADM1 to regulate STAT3 pathway activity and promote cell metastasis [58].

MiRNAs also regulate other proteins upstream of STAT3 signalling pathways. Kinesin family member 20A (KIF20A), a member of kinesin superfamily 6 , plays a significant role in the occurrence and development of tumours [59]. Functional experiments have shown that miR-876-3p inhibits cell proliferation, migration, and invasion. MiR-876-3p also acts as a tumour suppressor in a nude mouse model. Mechanistic experiments have proven that miR-876-3p inhibits the JAK2/STAT3 signalling pathway by targeting KIF20A [60]. AP-2 $\alpha$, a central member of the AP-2 family, can function as a tumour suppressor to block the IL-6/Jak2/STAT3 signalling pathway [61]. MiR-26a, which functions as an oncogene, binds to the 3'-UTR of AP- $2 \alpha$ and then inhibits AP- $2 \alpha$ expression to promote glioma cell chemoresistance, migration, invasion, and proliferation [62]. MiR-6743-5p regulates the activity of STAT3 and promotes cell proliferation by directly targeting a gene associated with retinoid interferon-induced mortality-19 (GRIM-19), which serves as a tumour suppressor [63].

Exosomes, containing mRNAs, microRNAs, and long noncoding RNAs, deliver their contents to recipient cells and function as regulators of intercellular communication [64]. Based on RT-qPCR assays, hypoxic glioma-derived exosomes (H-GDEs) and the CSF of GBM patients had a significantly higher expression level of miR-1246 than normoxic glioma-derived exosomes (N-GDEs) and the CSF of low-grade glioma (LGG) 
patients. H-GDE-derived miR-1246 contributed to M2 macrophage polarization, which then promoted glioma progression in vivo and promoted glioma cell migration, invasion, and proliferation in vitro. Regarding the mechanism by which H-GDE-derived miR-1246 induced M2 macrophage polarization, it was found that it targeted telomeric repeat binding factor 2 interacting protein (TERF2IP) and suppressed TERF2IP expression to activate the STAT3 signalling pathway and inhibit NF-kB signalling in macrophages. In summary, H-GDE miR-1246 plays an important role in M2 macrophage polarization [65]. GBM can be subclassified into three clinically relevant types: proneural (PN), neural, mesenchymal (MES) and classical GBM [66]. MiRNAs are also involved in the process of maintaining the phenotype of GBM. A study found that the activated wnt/b-catenin signalling pathway can increase the expression of miR-125b and miR-20b, which then inhibit the expression of frizzled receptor 6 (FZD6) and ALDH1A3 to sustain Wnt/bcatenin signalling in PN GBM. However, in MES GBM, FZD6 downregulated the canonical wnt/b-catenin signalling pathway by activating the CaMKII-TAK1-NLK pathway, which can promote the STAT3 and NF-kB signalling pathways [67].

\section{MiRNAs regulated by STAT3}

As a member of the STAT protein family, STAT3 acts as an important transcription factor, which can initiate the transcription of downstream target genes. For example, chromatin immunoprecipitation (ChIP) analysis has shown that STAT3 binds to the miR-21 promoter region to regulate the expression of miR-21; thus, IFN- $\beta$ can downregulate miR-21 transcription [68]. There is a feedback loop in which miR-21 can suppress both PIAS3 protein and mRNA expression to activate STAT3 in medulloblastoma [69]. RT-PCR experiments have indicated that the expression of miR-21 increased with glioma pathological grade. Studies have found that miR21 can target reversion-inducing cysteine-rich protein with kazal motifs (RECK) to regulate glioma cell invasion and the expression of MMP2/9 [70]. Additionally, miR-21 promoted glioma cell growth and the expression of human telomerase reverse transcriptase (hTERT) in a STAT3-dependent manner [71]. Moreover, the expression of miR-182-5p was markedly increased and positively correlated with the expression of activated STAT3 in glioma cell lines. Functional experiments have shown that miR-182-5p promotes glioma cell growth, migration, and invasion. In terms of the mechanism, STAT3 can directly interact with the miR-182-5p promoter region, and miR-182-5p can directly target sequences in the 3'-UTR of protocadherin 8 (PCDH8) [72]. In addition, in hypoxic extracellular vesicles (EVs) of glioma stem-like cells (GSCs), RT-PCR assays have shown that miR-30b-3p is expressed at high levels. Studies have found that miR-30b-3p can bind to sites on the $3^{\prime}$-UTR of ras homologue family member B (RHOB), decrease cell cycle arrest and inhibit cell apoptosis induced by TMZ. As a translation factor, STAT3 can bind to the miR-30b promoter, but STAT3 must form a complex with HIF $1 \alpha$ to induce miR-30b-3p expression in GSCs under hypoxic conditions [73]. In contrast, STAT3 can inhibit the expression of some miRNAs. Upon activation of RTK signalling, STAT3 directly interacts with the miR-218 locus and then inhibits its expression. However, miR-218, acting as a tumour suppressor, can directly interact with platelet-derived growth factor receptor $\alpha$ (PDGFR $\alpha$ ) to inhibit the activity of RTK signalling. In summary, activated RTK signalling increases the expression of STAT3 and then represses miR-218 expression [74]. Additionally, STAT3 can bind to the first intron of the miR-34a genomic region to suppress miR-34a expression, while miR-34a acts as a tumour suppressor in hypoxia-induced glioma cells [75].

\section{Other miRNAs}

In addition to the above three miRNAs, other miRNAs can also affect the expression of STAT3, but the specific mechanism has not yet been elucidated. A study has shown that overexpression of miR-184 promotes cell proliferation and invasion through the JAK2/STAT3 signalling pathway [76]. Moreover, the overexpression of miR-203 increased malignant glioma (MG) cell radiosensitivity by downregulating the expression of the JAK/ STAT3 pathway [77]. RT-qPCR assays revealed that miR-31 expression was significantly decreased in U87 and U251 cells. Overexpression of miR-31 induces GBM cell apoptosis and suppresses the activity of STAT3 [78]. MiR-27b, possibly mediated by the $\beta$-catenin/Tcf- 4 pathway, can promote glioma cell proliferation and invasion and decrease the cell apoptosis rate [79]. In extensively mutated U87MG, miR-302/367 overexpression can clearly suppress the expression of transformation-related proteins, including STAT3 signalling [80].

In summary, some miRNAs serve as target genes and are transcriptionally regulated by STAT3. Meanwhile, to regulate the expression and activation of STAT3 in glioma, the main mechanism underlying miRNA function is through direct binding of the $3^{\prime}$-UTR of the target mRNA. In addition to the traditional mechanism, miRNAs can be present in exosomes to induce M2 macrophage polarization and maintain the GBM phenotype. However, some miRNAs need more in-depth study to uncover their specific mechanism. In addition, studies should focus on investigating other mechanisms of miRNA function. MiRNAs can bind to the $5^{\prime}$-UTR of the 
target gene and promote its transcription. For example, a study showed that miR-27a-3p could act as an oncogene by directly interacting with the 5'-UTR of cyclin D1 to enhance its expression in bladder cancer [81].

\section{LncRNAs are involved in the STAT3 signalling pathway}

Long noncoding RNAs, which are more than 200 nucleotides in length, generally have no protein coding ability or can only code a few short peptides. LncRNAs are widespread in human organisms and are essential for human gene expression regulation and physiological and pathological processes. Most types of lncRNAs are transcribed by RNA polymerase II and are then capped at the 5 ' end, polyadenylated at the 3 ' end and edited through a series of splicing processes that occur in the nucleus. In addition, there are other mechanisms involved in the lncRNA maturation process. According to the position and directional relationship with the gene encoding protein, lncRNAs can be divided into five categories: sense lncRNAs, antisense lncRNAs, intronic lncRNAs, bidirectional lncRNAs, and intergenic lncRNAs [82]. In terms of the multiple functions of IncRNAs, in the cytoplasm, the most common function of lncRNAs is their ability to act as competing endogenous RNAs (ceRNAs), and lncRNAs can regulate translation, serve as protein scaffolds and transcribe short peptides [83]. In the nucleus, lncRNAs can interact with transcription factors and suppress gene transcription [84]. Similar to miRNAs, lncRNAs are also cell-specific and sequentially expressed. However, unlike miRNAs, lncRNA sequences are less conserved, and lncRNAs can fully regulate gene expression at the epigenetic level, transcription level and posttranscriptional level (Fig. 2).

Numerous studies have proven that there are a large number of abnormally expressed lncRNAs in gliomas, and they play a vital role in the occurrence and

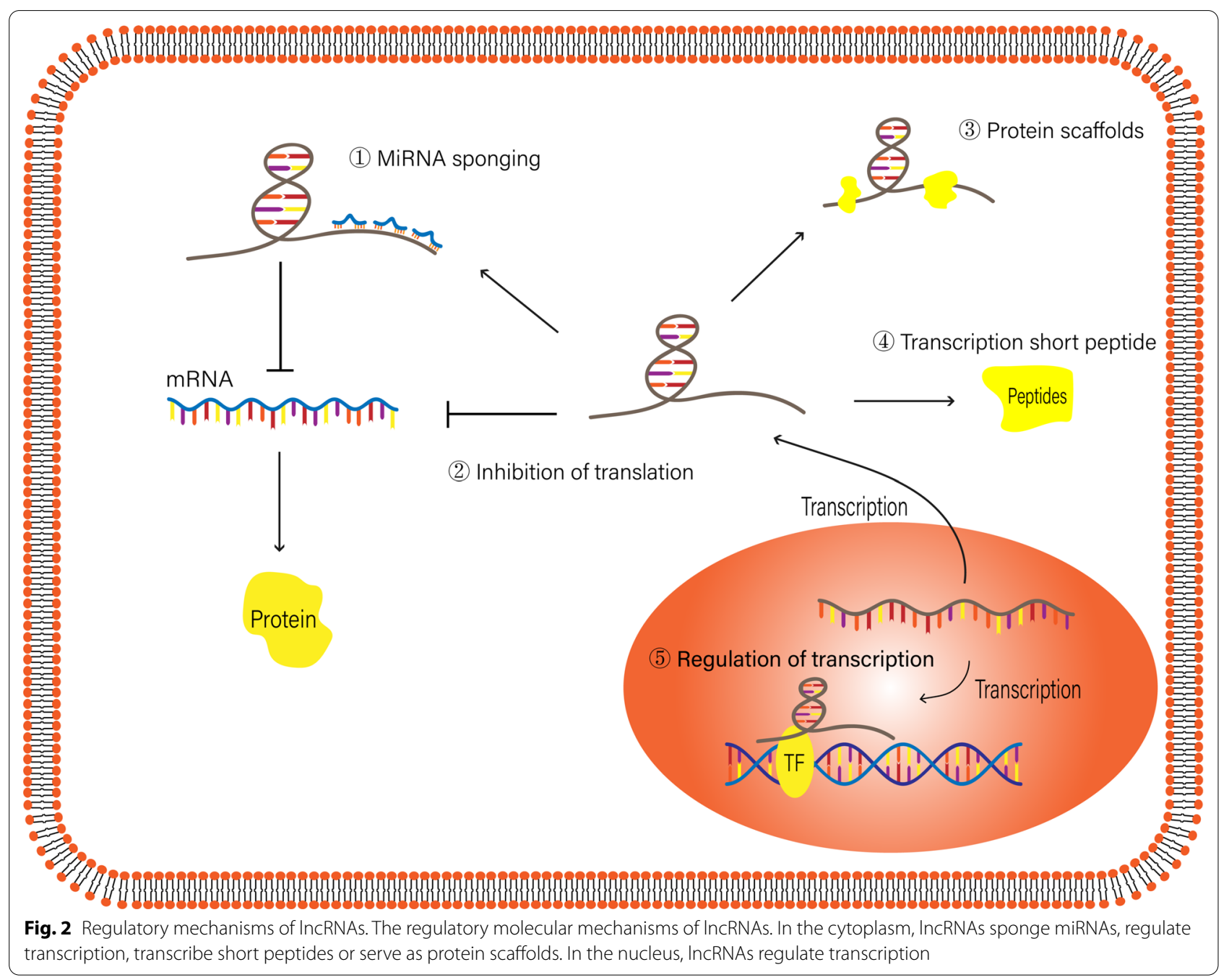


development of gliomas. The abnormal expression and role of these lncRNAs in gliomas makes them promising targets for the early diagnosis and treatment of gliomas. LncRNAs regulate the expression and activation of various signalling pathways and target genes in gliomas [85]. Among them, lncRNAs related to STAT3 are mainly divided into the following types as shown in Table 2.

\section{LncRNAs regulate the expression and activation of STAT3 through the ceRNA mechanism}

Their ability to act as competing endogenous RNAs (ceRNAs) or miRNA sponges is the most common function of lncRNAs. The lncRNAs located in the cytoplasm likely regulate the expression of downstream genes through this ceRNA mechanism. The expression of lncRNA CASC9 (cancer susceptibility candidate 9) is significantly increased in glioma tissue and is positively related to advanced pathological grade of glioma. Knocking down lncRNA CASC9 inhibits glioma cell invasion and proliferation. In terms of the mechanism responsible for this effect, the 3'-UTR of CASC9 can interact with miR-519d, which acts as a tumour suppressor by directly targeting the 3'-UTR of STAT3 mRNA, to increase the expression of STAT3. Moreover, ChIP and luciferase reporter assays demonstrated that STAT3 can increase the expression of CASC9 by binding to the promoter of CASC9. Taken together, in glioma, IncRNA CASC9 increases STAT3 expression by sponging miR-519d, while STAT3 interacts with the CASC9 promoter to accelerate CASC9 expression [86].

\section{LncRNAs regulate the expression of genes upstream of STAT3}

The activation of STAT3 is precisely regulated. In normal cells, the activation of STAT3 is short-lived, while in glioma cells, STAT3 exhibits constitutively high activation and participates in the occurrence and development of tumours due to the lack of upstream regulatory signals. Similar to miRNAs, lncRNAs can also increase the expression and activity level of STAT3 by regulating its upstream regulatory signals. Coimmunoprecipitation and immunoprecipitation experiments have shown that piwilike RNA-mediated gene silencing 4 (PIWIL4), belonging to the PIWI subfamily, could induce activation of STAT3. The expression of colorectal neoplasia differentially expressed (CRNDE) is high in glioma. Knockdown of CRNDE induced glioma cell apoptosis, while overexpression of CRNDE promoted U87 and U251 cell proliferation, migration and invasion. RT-PCR assays showed that the expression of miR-384 was decreased in glioma tissues and glioma cell lines and negatively correlated with advanced glioma pathological grade. Luciferase assays showed that CRNDE could bind to miR-384, while miR384 could target PIWIL4 to function as a tumour suppressor. LncRNA CRNDE increased PIWIL4 expression to induce p-STAT3 through a ceRNA mechanism [87]. In addition, Kaplan-Meier survival analysis and datasets showed that the expression level of LINC00115, which is activated by TGF- $\beta$, was higher in GBM than in LGG and correlated with a statistically poor prognosis. In vitro experiments found that knockdown of LINC00115 inhibited GSC proliferation and neuro-like sphere formation. In vivo experiments found that LINC00115 knockdown reduced glioma tumour growth and prolonged the survival of animals. LINC00115 can physically associate with miR-200b and miR-200c to competitively inhibit the function of miR-200b and miR-200c. MiR-200b and miR-200c can bind to the $5^{\prime}$-UTR of zinc finger protein 596 (ZNF596) mRNA. Meanwhile, ChIP-qPCR and luciferase reporter analysis demonstrated that ZNF596 could directly bind with the promoter of enhancer of zeste homologue 2 (EZH2), which is a lysine methyltransferase and the enzymatic component of the polycomb repressive complex 2. Due to EZH2 promoting histone H3 lysine 27 trimethylation (H3K27me3) and activating STAT3 signalling, LINC00115, acting as an oncogene, competitively binds with miR-200 s and activates ZEB1 signalling, and ZNF596 enhances EZH2/STAT3 signalling [88]. Moreover, based on three public human astrocytoma databases (TCGA, CGGA and Rembrandt), the

Table 2. LncRNAs/CircRNAs involved in STAT3 signaling pathway

\begin{tabular}{llllll}
\hline LncRNA/CircRNA & $\begin{array}{l}\text { Up-/down- } \\
\text { regulation }\end{array}$ & Role in glioma & Biological functions & Target gene & Refs. \\
\hline CASC9 & Up & Oncogene & Promote invasion and proliferation & miR-519d/STAT3 \\
CRNDE & Up & Oncogene & Promote proliferation, migration, and invasion & miR-384/PIWIL4 \\
LINC00115 & Up & Oncogene & Promote proliferation & miR-200b, miR-200c/ZNF596 & [88] \\
miR155HG & Up & Oncogene & Promote proliferation & miR-185-5p/ANXA2 \\
PEG10 & Up & Oncogene & Promote proliferation, migration, and invasion & miR-506 & [89] \\
GHET1 & Up & Oncogene & Promote proliferation, migration, and invasion & miR-216a & [90] \\
Circ-HIPK3 & Up & Oncogene & Promote proliferation, migration, and invasion & miR-124-3p/STAT3 & [91] \\
\hline
\end{tabular}


expression of the miR155 host gene (miR155HG) is high in GBM tissues and positively associated with the expression of genes that reduce apoptosis and cell death. The GBM mouse model found that miR155HG increased tumour volume. In vitro, miR155HG silencing inhibited cell proliferation and increased cell apoptosis. These results show that miR155HG is an oncogene in GBM. In terms of the mechanism, miR155HG can bind with miR185-5p and then sponge miR-185-5p to downregulate its expression. MiR-185-5p directly targets the $3^{\prime}$-UTR of ANXA2, which can act as an oncogene by regulating the expression of p-STAT3. Additionally, ChIP and luciferase reporter gene assays showed that p-STAT3 could bind to the promoter region of miR155HG to increase the expression of miR155HG. In summary, the authors established the miR155HG/miR-185-5p/ANXA2 loop in GBM [89].

\section{LncRNAs regulated by STAT3}

Similar to miRNAs, lncRNAs can also serve as target genes of STAT3 in gliomas and undergo transcriptional regulation. Previous studies have shown that STAT3 interacts with the promoter region of CASC9, while CASC9 competitively binds with miR-519d to increase the expression of STAT3. Similarly, p-STAT3 directly binds to the miR155HG promoter region. MiR155HG inhibits miR-185-5p expression through a ceRNA mechanism and then promotes ANXA2 expression to accelerate the activation of STAT3.

LncRNAs regulate STAT3 through an unknown mechanism In addition, some lncRNAs can affect the expression of STAT3 without a clear and specific mechanism. For example, knockdown of lncRNA PEG10 inhibited U251 cell proliferation, migration, and invasion and inactivated the Raf/MEK/ERK and JAK1/STAT3 signalling pathways by increasing miR-506 expression [90]. In addition, lncRNA gastric carcinoma highly expressed transcript 1 (GHET1), acting as an oncogene; promoted cell proliferation, migration, and invasion; and activated the JAK2/ STAT3 and p53/survivin signalling pathways by miR216a downregulation [91].

In summary, although IncRNA PEG10 and GHET1 require future studies to reveal the specific mechanism, it is clear that the ceRNA mechanism plays an important role in the regulation of the STAT3 pathway by lncRNAs. Moreover, studies established 2 positive feedback loops: the miR155HG/miR-185-5p/ANXA2 loop and the CASC9/miR-519d/STAT3 loop. Regarding transcription factor research in glioma formation and progression, future studies can also try to verify the existence of such a positive feedback loop. For instance, Lin and colleagues, found that the positive feedback loop HOXC-AS2/
miR-876-5p/ZEB1 could regulate epithelial-mesenchymal transition (EMT) in GBM [92]. In addition to the ceRNA mechanism, research should focus on additional mechanisms. In glioma, lncRNA PLAC2 negatively regulates RPL36 expression levels through the STAT1 signalling mechanism. In the nucleus, PLAC2 can bind with STAT1 and then interact with the promoters of RPL36, while cytoplasmic PLAC2 can also inhibit STAT1 translocation into the nucleus [93].

\section{CircRNAs are involved in the STAT3 signalling pathway}

Following the investigation of miRNAs and lncRNAs, circRNAs have become a new research focus. Unlike linear RNA, circRNAs have a covalent single-stranded closed loop structure, lacking a 5' cap and 3' tail, and the structure of circRNA is highly stable compared with linear RNA. According to the various positions and formation mechanisms of circRNAs, they can be divided into three subclasses: exonic circRNAs, intronic circRNAs and EIciRNAs [94]. It has been found that more than $80 \%$ of circRNAs are derived from protein-coding gene exons in the cytoplasm. In the past, it was thought that circRNAs were a by-product of transcription, had low expression abundance and were non-functional. However, with the development of high-throughput sequencing and RNA detection technology, a large number of circRNAs, which are produced by back-splicing of a pre-mRNA, have been found in normal tissues and different tumours. The function and molecular mechanism of circRNAs have also been revealed. Studies have found that circRNAs work through various mechanisms. In the cytoplasm, circRNAs can regulate miRNAs through ceRNA mechanisms. CircRNAs can also form circRNA-protein complexes (circRNPs) by binding to proteins, thereby regulating the function of related proteins, intracellular localization and transcription of related genes. Some circRNAs can transcribe short peptides or serve as protein scaffolds [95, 96]. In the nucleus, circRNAs can regulate the transcription and splicing of genes [97] (Fig. 3).

The expression of circRNAs is tissue specific and developmental stage dependent. Many studies have shown that abnormally expressed circRNAs are involved in the pathophysiological process of glioma. For example, the circRNA HIPK3 [98] regulates the proliferation, migration, and invasion of tumour cells through a ceRNA mechanism. Circ-DICER1 [99] is involved in the angiogenesis of glioma. Circ_0076248 [100] is involved in the immune response of glioma. Circ_0001649 can be used as a diagnostic and prognostic indicator [101].

There is only one study on the role of circRNA in regulating the STAT3 pathway. Based on qRT-PCR assays, the expression of the circular RNA HIPK3 was significantly 


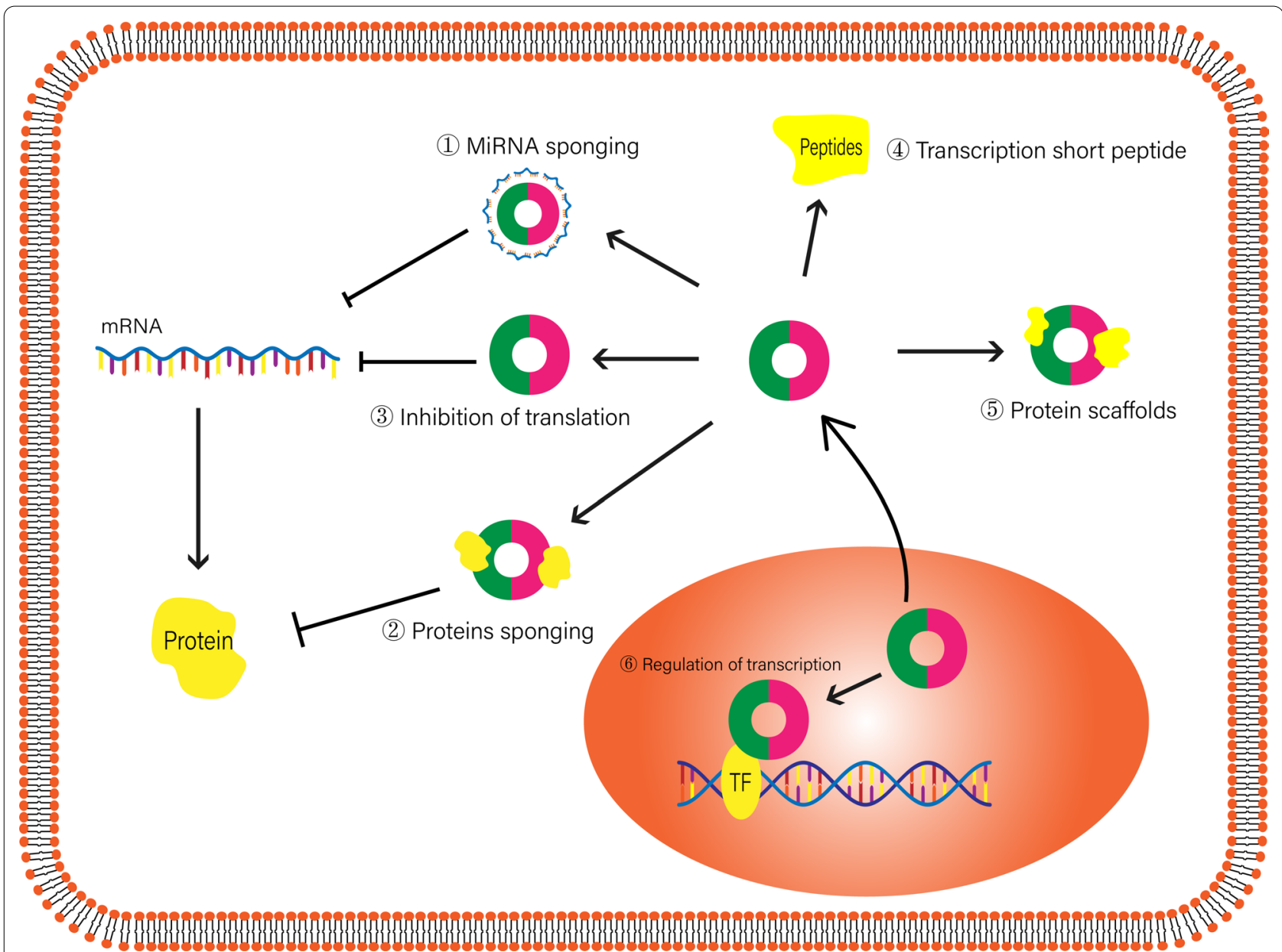

Fig. 3 Regulatory mechanisms of circRNAs. The regulatory molecular mechanisms of circRNAs. In the cytoplasm, circRNAs sponge miRNAs and proteins. CircRNAs regularly transcribe short peptides or serve as protein scaffolds. In the nucleus, circRNAs regulate transcription

higher in the U251 and U87 cell lines than in the normal cell line (HEB), while miR-124-3p was expressed at a low level in the U251 and U87 cell lines. Functional experiments confirmed that circ-HIPK3 knockdown by targeted siRNAs inhibited cell proliferation, migration, and invasion and induced cell apoptosis. Mechanistic experiments have shown that circ-HIPK3 directly binds to miR-124-3p and that miR-124-3p can directly target the 3'-UTR of STAT3 mRNA. In summary, circ-HIPK3 suppresses the mRNA expression of miR-124-3p, an inhibitor of STAT3, through a ceRNA mechanism. Due to the low expression of miR-124-3p, STAT3 expression is increased [102].

In summary, studies should investigate more circRNAs involved in the glioma STAT3 signalling pathway. Mechanistically, similar to lncRNAs, the ceRNA mechanism is the most common functional mechanism of circRNas, while few studies have found that circRNAs can induce STAT3 nuclear translocation. In colorectal cancer,
circSPARC not only regulates the expression of JAK2 through the ceRNA mechanism to affect the activation of STAT3 but also enhances the nuclear translocation of p-STAT3 through the recruitment of FUS RNA binding protein [103].

\section{Conclusions}

In summary, an increasing number of studies have shown that noncoding RNAs play an important role in a variety of malignant tumours. The role and mechanism of noncoding RNAs in glioma has been a research hotspot in recent years, and they are expected to become diagnostic and prognostic markers and therapeutic targets for glioma. Moreover, the continuous high expression and activation of STAT3 is an important factor in the occurrence and development of glioma. STAT3 is considered a potential therapeutic target for glioma, but there is no effective targeted STAT3 clinical treatment method for glioma. In this review, we analysed the noncoding RNAs 
that regulates the expression of STAT3 and that are regulated by STAT3 in gliomas (Fig. 4).

In terms of STAT3, as a transcription factor, there is no doubt that STAT3 can play an important role in regulating the expression of ncRNAs. LncRNAs CASC9 and miR155HG promote STAT3 through the ceRNA mechanism, and STAT3, in turn, acts as a transcription factor in the nucleus of glioma cells to promote the expression of CASC9 and miR155HG. These molecules form a positive feedback loop to promote their expression. For miRNAs, activated STAT3 can promote the expression of many oncogenic miRNAs, such as miR-182-5p, miR21 and miR-30b-3p. Similarly, STAT3 can also inhibit the expression of miR-218, a tumour suppressor. In terms of miRNAs, the most common mechanism of miRNA function is binding to the $3^{\prime}$-UTR of the target mRNA. In the STAT3 signalling pathway of glioma, miRNAs can directly target STAT3 mRNA, genes closely related to STAT3 activation (PIAS3, SOCS3), and upstream genes of STAT3. In addition, miRNAs, such as miR-1246, can also play a role by being packaged inside exosomes. However, some miRNAs can regulate the expression and activation of STAT3, but the specific mechanism is still not clear; thus, it is necessary to explore these specific mechanisms. In terms of lncRNAs and circRNAs, these RNAs can regulate the expression of target genes

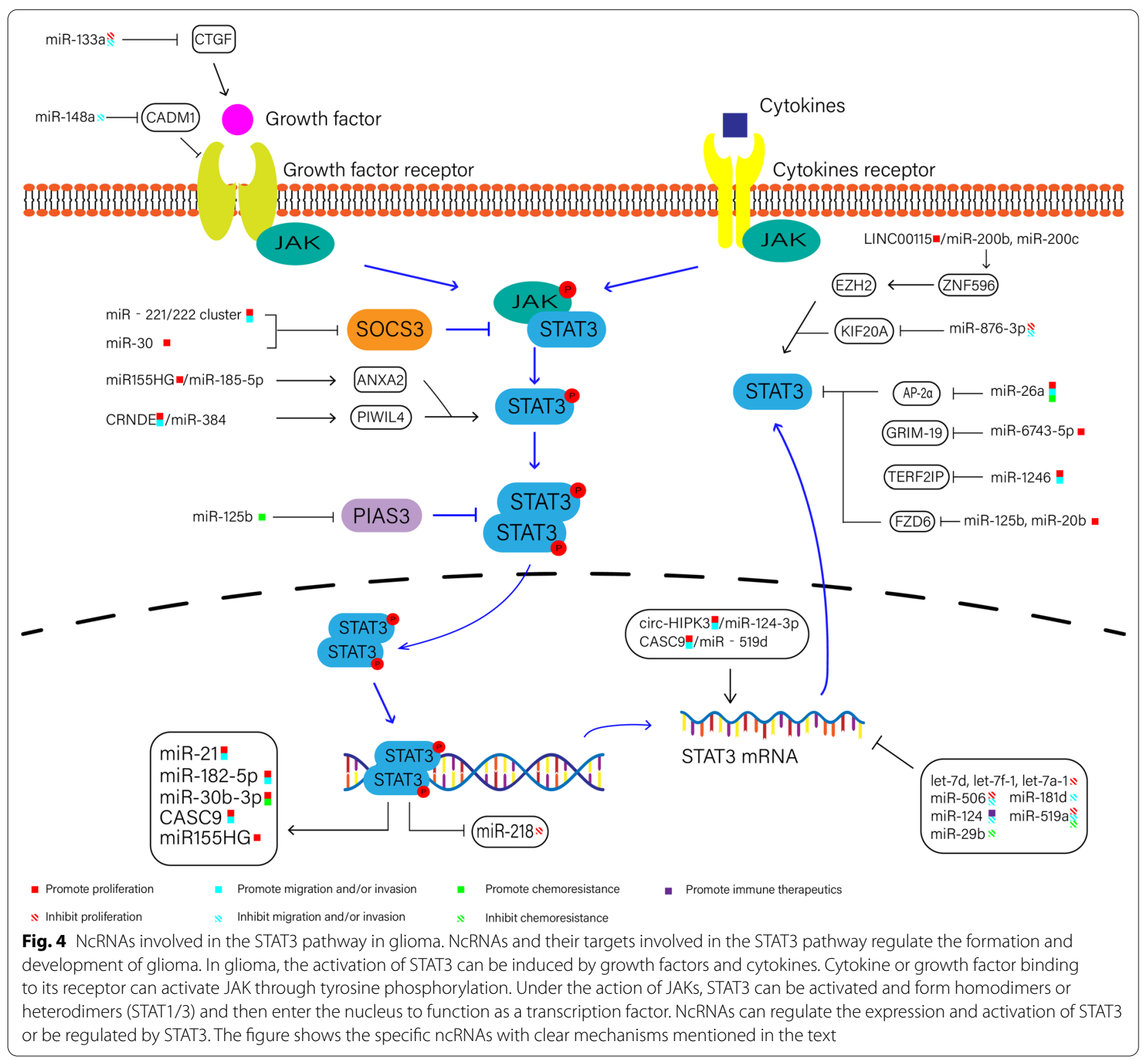


at the epigenetic, transcriptional and posttranscriptional level. However, studies have mainly focused on lncRNAs and circRNAs that regulate the transcription level of STAT3 through the ceRNA mechanism. In addition to the ceRNA mechanism, other mechanisms of lncRNAs and circRNAs have been reported. For example, in nasopharyngeal carcinoma, IncRNA differentiation antagonizing nonprotein coding RNA (DANCR) can interact with STAT3 to enhance JAK1 binding to STAT3 [104]. LncRNA LOC101927514 was detected in the nucleus where it interacted with STAT3, thereby participating in the release of IL-6 and IL-8 [105]. Circ-Amotll not only interacts with STAT3 to increase STAT3 expression but also promotes STAT3 nuclear translocation [106]. Therefore, in addition to ceRNA mechanisms, studies should explore other mechanisms by which lncRNAs and circRNAs regulate the STAT3 signalling pathway in gliomas.

In recent years, research on tumour immunity has gained more attention. Interferon, as an immune-related cytokine, can play an important role in the antitumour process, and interferons play important roles in STAT3 activation. Of course, some ncRNAs are also involved in this process. In glioma, IFN- $\beta$ regulates miR-21 through STAT3, which is an interesting finding. IFN $-\beta$ can induce STAT3 activation, but the role of STAT3 is distinct. The p-STAT3 induced by IFN- $\beta$ can directly bind to the promoter of miR-21 and then inhibit the transcription of this carcinogenic miRNA, but STAT3 promotes the transcription of miR-21 without IFN- $\beta$ [68]. Different cytokine stimuli and cell types may contribute to the controversial role of STAT3. Although there is no research on the lncRNAs and circRNAs involved in this process of glioma, their mechanisms are different from those of miRNAs. In the cytoplasm of hepatocellular carcinoma cells, LncRNA00364, which is upregulated by IFN- $\gamma$ can directly interact with STAT3 and then inhibit the phosphorylation of tyrosine-705 of STAT3 to function as a tumour suppressor [107]. In addition, immune cells involved in the tumour microenvironment also play important roles in tumour immunity. Some ncRNAs are involved in the STAT3 signalling pathway of immune cells. M2 polarization macrophages make up a majority of glioma tumour-associated macrophages and help create a tumour-immunosuppressive microenvironment to promote tumour function [108]. To induce M2 macrophage polarization, H-GDE-derived miR-1246 can inhibit TERF2IP expression and then activate the STAT3 signalling pathway in macrophages [65]. Contrary to M2 macrophages, $\mathrm{T}$ cells can promote tumour suppression. In glioma, miR-124 can enhance $T$ cell-mediated immune clearance and modulate $\mathrm{T}$ helper cell differentiation by inhibiting the STAT3 signalling pathway [48]. As the literature has not revealed relevant findings, glioma-related studies should focus more attention on lncRNAs and circRNAs involved in the STAT3 signalling pathway of immune cells. MHC class I-related chain (MIC)-A, which is a downstream target of STAT3, can activate natural killer $\mathrm{T}$ (NKT) cells. In cervical cancer, a study has shown that LINC00240 can promote STAT3 expression by sponging miR-124-3p, and then STAT3 can inhibit MICA to reduce the cytotoxicity of NKT cells [109]. Overall STAT3 plays a vital role in tumour immunity. To reveal the role of STAT3 in glioma, future studies should focus on the ncRNAs involved in these two processes.

Therefore, further exploration of noncoding RNAs related to STAT3 expression and activation in glioma may provide an avenue for STAT3-targeted glioma treatment.

\section{Acknowledgements}

None.

\section{Authors' contributions}

JS and XZ provided ideas for the manuscript. BX, WJ and BX wrote the manuscript. $\mathrm{ZH}$ and $\mathrm{HH}$ provided the figures. JH provided the tables. JJ edited the manuscript. All authors read and approved the final manuscript.

\section{Funding}

This work was supported by the National Natural Science Foundation of China (81672487), the Jiangsu Young Medical Talents (QNRC2016190), and the Medical Talents of Wuxi People's Hospital (RKA20181).

Availability of data and materials

Not applicable.

\section{Declarations}

Ethics approval and consent to participate Not applicable.

Consent for publication

We agree to publish the manuscript.

Competing interests

The authors have no conflicts of interest to declare.

Received: 24 April 2021 Accepted: 11 August 2021

Published online: 23 August 2021

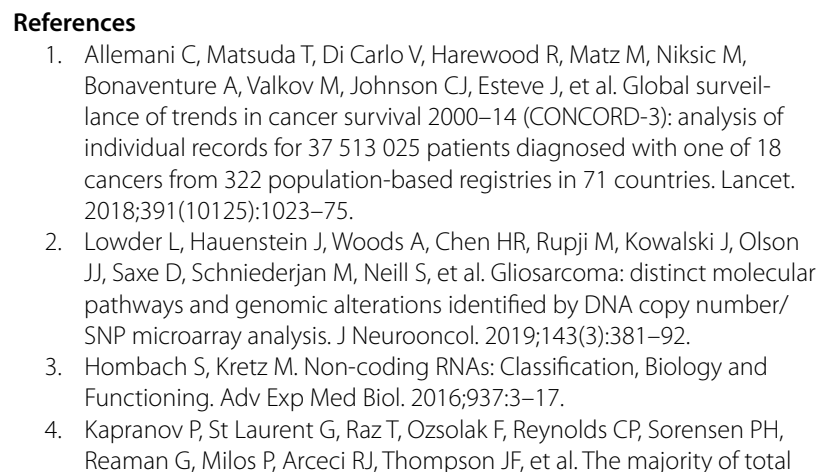

References Bonaventure A, Valkov M, Johnson CJ, Esteve J, et al. Global surveilance of trends in cancer survival 2000-14 (CONCORD-3): analysis of dividual records for 37513025 patients diagnosed with one of 18 cancers from 322 population-based registries in 71 countries. Lancet. 2018:391(10125):1023-75.

$J$ J, Saxe D, Schniederjan M, Neill S, et al. Gliosarcoma: distinct molecular pathways and genomic alterations identified by DNA copy number/ microarray analysis. J Neurooncol. 2019:143(3):381-92.

Functioning. Adv Exp Med Biol. 2016:937:3-17.

Reaman G, Milos P, Arceci RJ, Thompson JF, et al. The majority of total 
nuclear-encoded non-ribosomal RNA in a human cell is "dark matter" un-annotated RNA. BMC Biol. 2010;8:149.

5. Panni S, Lovering RC, Porras P, Orchard S. Non-coding RNA regulatory networks. Biochim Biophys Acta Gene Regul Mech. 2020;1863(6):194417.

6. Chan JJ, Tay Y. Noncoding RNA:RNA Regulatory Networks in Cancer. Int J Mol Sci. 2018;19:5.

7. Song J, Kim YK. Targeting non-coding RNAs for the treatment of retinal diseases. Mol Ther Nucleic Acids. 2021;24:284-93.

8. Ji W, Jiao J, Cheng C, Shao J. MicroRNA-21 in the pathogenesis of traumatic brain injury. Neurochem Res. 2018;43(10):1863-8.

9. Ouedraogo ZG, Biau J, Kemeny JL, Morel L, Verrelle P, Chautard E. Role of STAT3 in genesis and progression of human malignant gliomas. Mol Neurobiol. 2017:54(8):5780-97.

10. Lo HW, Cao X, Zhu H, Ali-Osman F. Constitutively activated STAT3 frequently coexpresses with epidermal growth factor receptor in high-grade gliomas and targeting STAT3 sensitizes them to Iressa and alkylators. Clin Cancer Res. 2008;14(19):6042-54.

11. Buettner R, Mora LB, Jove R. Activated STAT signaling in human tumors provides novel molecular targets for therapeutic intervention. Clin Cancer Res. 2002;8(4):945-54.

12. Swiatek-Machado K, Kaminska B. STAT signaling in glioma cells. Adv Exp Med Biol. 2013;986:189-208.

13. Lindemann C, Hackmann O, Delic S, Schmidt N, Reifenberger G, Riemenschneider MJAn: SOCS3 promoter methylation is mutually exclusive to EGFR amplification in gliomas and promotes glioma cell invasion through STAT3 and FAK activation. 2011, 122(2):241-251.

14. Brantley EC, Nabors LB, Gillespie GY, Choi YH, Palmer CA, Harrison K, Roarty K, Benveniste EN. Loss of protein inhibitors of activated STAT-3 expression in glioblastoma multiforme tumors: implications for STAT-3 activation and gene expression. Clin Cancer Res. 2008:14(15):4694-704.

15. Shi Y, Guryanova OA, Zhou W, Liu C, Huang Z, Fang X, Wang X, Chen C, Wu Q, He Z, et al. Ibrutinib inactivates BMX-STAT3 in glioma stem cells to impair malignant growth and radioresistance. Sci Transl Med. 2018:10:443.

16. Sun X, Wang J, Huang M, Chen T, Chen J, Zhang F, Zeng H, Xu Z, Ke Y. STAT3 promotes tumour progression in glioma by inducing FOXP1 transcription. J Cell Mol Med. 2018;22(11):5629-38.

17. Zhang L, Li Y, Liu Y, Wang $X$, Chen M, Xing Y, Zhu D. STAT3-mediated MMP-2 expression is required for 15-HETE-induced vascular adventitial fibroblast migration. J Steroid Biochem Mol Biol. 2015;149:106-17.

18. Kang SH, Yu MO, Park KJ, Chi SG, Park DH, Chung YG. Activated STAT3 regulates hypoxia-induced angiogenesis and cell migration in human glioblastoma. Neurosurgery. 2010;67(5):1386-95.

19. Yang XO, Panopoulos AD, Nurieva R, Chang SH, Wang D, Watowich SS, Dong C. STAT3 regulates cytokine-mediated generation of inflammatory helper T cells. J Biol Chem. 2007;282(13):9358-63.

20. Zhang M, Zhou L, Xu Y, Yang M, Xu Y, Komaniecki GP, Kosciuk T, Chen X, LuX, Zou X, et al. A STAT3 palmitoylation cycle promotes TH17 differentiation and colitis. Nature. 2020;586(7829):434-9.

21. Yuan X, Du J, Hua S, Zhang H, Gu C, Wang J, Yang L, Huang J, Yu $J$, Liu F. Suppression of autophagy augments the radiosensitizing effects of STAT3 inhibition on human glioma cells. Exp Cell Res. 2015;330(2):267-76

22. Tan MSY, Sandanaraj E, Chong YK, Lim SW, Koh LWH, Ng WH, Tan NS, Tan P, Ang BT, Tang C. A STAT3-based gene signature stratifies glioma patients for targeted therapy. Nat Commun. 2019;10(1):3601.

23. Abreu FB, Liu X, Tsongalis GJ. miRNA analysis in pancreatic cancer: the Dartmouth experience. Clin Chem Lab Med. 2017;55(5):755-62.

24. Lee Y, Kim M, Han J, Yeom KH, Lee S, Baek SH, Kim VN. MicroRNA genes are transcribed by RNA polymerase II. EMBO J. 2004;23(20):4051-60.

25. Lee Y, Ahn C, Han J, Choi H, Kim J, Yim J, Lee J, Provost P, Radmark O, Kim S, et al. The nuclear RNase III Drosha initiates microRNA processing. Nature 2003:425(6956):415-9.

26. Wang J, Lee JE, Riemondy K, Yu Y, Marquez SM, Lai EC, Yi R. XPO5 promotes primary miRNA processing independently of RanGTP. Nat Commun. 2020;11(1):1845.

27. Saliminejad K, Khorram Khorshid HR, Soleymani Fard S, Ghaffari SH. An overview of microRNAs: Biology, functions, therapeutics, and analysis methods. J Cell Physiol. 2019;234(5):5451-65.
28. Achkar NP, Cambiagno DA, Manavella PA. miRNA Biogenesis: A Dynamic Pathway. Trends Plant Sci. 2016;21(12):1034-44.

29. Olejniczak M, Kotowska-Zimmer A, Krzyzosiak W. Stress-induced changes in miRNA biogenesis and functioning. Cell Mol Life Sci. 2018;75(2):177-91.

30. Kabekkodu SP, Shukla V, Varghese VK, D'Souza J, Chakrabarty S, Satyamoorthy K. Clustered miRNAs and their role in biological functions and diseases. Biol Rev. 2018;93(4):1955-86.

31. Fabian MR, Sonenberg N, Filipowicz W. Regulation of mRNA translation and stability by microRNAs. Annu Rev Biochem. 2010;79:351-79.

32. Huntzinger E, Izaurralde E. Gene silencing by microRNAs: contributions of translational repression and mRNA decay. Nat Rev Genet. 2011;12(2):99-110.

33. Rouleau S, Glouzon JS, Brumwell A, Bisaillon M, Perreault JP. 3' UTR G-quadruplexes regulate miRNA binding. RNA. 2017;23(8):1172-9.

34. Xu H, Zhao G, Zhang Y, Jiang H, Wang W, Zhao D, Hong J, Yu H, Qi L. Mesenchymal stem cell-derived exosomal microRNA-133b suppresses glioma progression via Wnt/beta-catenin signaling pathway by targeting EZH2. Stem Cell Res Ther. 2019;10(1):381.

35. Wang S, Yin Y, Liu S. Roles of microRNAs during glioma tumorigenesis and progression. Histol Histopathol. 2019;34(3):213-22.

36. Ye X, Wei W, Zhang Z, He C, Yang R, Zhang J, Wu Z, Huang Q, Jiang Q. Identification of microRNAs associated with glioma diagnosis and prognosis. Oncotarget. 2017:8(16):26394-403.

37. Ji W, Jiao J, Cheng C, Xiao Y, Shao J, Liu H. A positive feedback loop of LINC00662 and STAT3 promotes malignant phenotype of glioma. Pathol Res Pract. 2021:224:153539.

38. Siddiqui WA, Ahad A, Ahsan H. The mystery of BCL2 family: BCl-2 proteins and apoptosis: an update. Arch Toxicol. 2015;89(3):289-317.

39. Doherty J, Baehrecke EH. Life, death and autophagy. Nat Cell Biol. 2018:20(10):1110-7.

40. Yang ZY, Wang Y, Liu Q, Wu M. microRNA cluster MC-let-7a-1 let-7d promotes autophagy and apoptosis of glioma cells by down-regulating STAT3. CNS Neurosci Ther. 2020;26(3):319-31.

41. Yu-Ju WuC, Chen CH, Lin CY, Feng LY, Lin YC, Wei KC, Huang CY, Fang $J Y$, Chen PY. CCL5 of glioma-associated microglia/macrophages regulates glioma migration and invasion via calcium-dependent matrix metalloproteinase 2. Neuro Oncol. 2020;22(2):253-66.

42. Peng T, Zhou L, Zuo L, Luan Y. MiR-506 functions as a tumor suppressor in glioma by targeting STAT3. Oncol Rep. 2016;35(2):1057-64.

43. Liu HW, Lee PM, Bamodu OA, Su YK, Fong IH, Yeh CT, Chien MH, Kan IH, Lin CM. Enhanced Hsa-miR-181d/p-STAT3 and Hsa-miR-181d/pSTAT5A Ratios Mediate the Anticancer Effect of Garcinol in STAT3/5AAddicted Glioblastoma. Cancers (Basel). 2019;11:12.

44. Choi S, Yu Y, Grimmer MR, Wahl M, Chang SM, Costello JF. Temozolomide-associated hypermutation in gliomas. Neuro Oncol. 2018:20(10):1300-9.

45. Xu JX, Yang Y, Zhang X, Luan XP. MicroRNA-29b promotes cell sensitivity to Temozolomide by targeting STAT3 in glioma. Eur Rev Med Pharmacol Sci. 2020;24(4):1922-31.

46. Hong L, Ya-Wei L, Hai W, Qiang Z, Jun-Jie L, Huang A, Song-Tao Q, Yun-Tao L. MiR-519a functions as a tumor suppressor in glioma by targeting the oncogenic STAT3 pathway. J Neurooncol. 2016;128(1):35-45.

47. Li H, Chen L, Li JJ, Zhou Q, Huang A, Liu WW, Wang K, Gao L, Qi ST, Lu YT. miR-519a enhances chemosensitivity and promotes autophagy in glioblastoma by targeting STAT3/BCl2 signaling pathway. J Hematol Oncol. 2018;11(1):70.

48. Wei J, Wang F, Kong LY, Xu S, Doucette T, Ferguson SD, Yang Y, McEnery K, Jethwa K, Gjyshi O, et al. miR-124 inhibits STAT3 signaling to enhance T cell-mediated immune clearance of glioma. Cancer Res. 2013;73(13):3913-26.

49. Li C, Li H, Zhang P, Yu LJ, Huang TM, Song X, Kong QY, Dong JL, Li PN, Liu J. SHP2, SOCS3 and PIAS3 Expression Patterns in Medulloblastomas: Relevance to STAT3 Activation and Resveratrol-Suppressed STAT3 Signaling. Nutrients. 2016;9:1.

50. Xu CH, Liu Y, Xiao LM, Chen LK, Zheng SY, Zeng EM, Li DH, Li YP. Silencing microRNA-221/222 cluster suppresses glioblastoma angiogenesis by suppressor of cytokine signaling-3-dependent JAK/STAT pathway. J Cell Physiol. 2019;234(12):22272-84. 
51. Che S, Sun T, Wang J, Jiao Y, Wang C, Meng Q, Qi W, Yan Z. miR-30 overexpression promotes glioma stem cells by regulating Jak/STAT3 signaling pathway. Tumour Biol. 2015;36(9):6805-11.

52. Jiao J, Zhang R, Li Z, Yin Y, Fang X, Ding X, Cai Y, Yang S, Mu H, Zong D, et al. Nuclear Smad6 promotes gliomagenesis by negatively regulating PIAS3-mediated STAT3 inhibition. Nat Commun. 2018;9(1):2504.

53. Shi L, Wan Y, Sun G, Zhang S, Wang Z, Zeng Y. miR-125b inhibitor may enhance the invasion-prevention activity of temozolomide in glioblastoma stem cells by targeting PIAS3. BioDrugs. 2014;28(1):41-54.

54. Song ZB, Yang HP, Xu AQ, Zhan ZM, Song Y, Li ZY. Connective tissue growth factor as an unfavorable prognostic marker promotes the proliferation, migration, and invasion of gliomas. Chin Med J (Engl). 2020;6:670-8

55. Zhang P, Chen FZ, Jia QB, Hu DF. Upregulation of microRNA-133a and downregulation of connective tissue growth factor suppress cell proliferation, migration, and invasion in human glioma through the JAK STAT signaling pathway. IUBMB Life. 2019;71(12):1857-75.

56. Zhang Z, Gong Q, Li M, Xu J, Zheng Y, Ge P, Chi G. MicroRNA-124 inhibits the proliferation of C 6 glioma cells by targeting Smad4. Int J Mol Med. 2017;40(4):1226-34.

57. Vallath S, Sage EK, Kolluri KK, Lourenco SN, Teixeira VS, Chimalapati S, George PJ, Janes SM, Giangreco A. CADM1 inhibits squamous cell carcinoma progression by reducing STAT3 activity. Sci Rep. 2016;6:24006.

58. Cai Q, Zhu A, Gong L. Exosomes of glioma cells deliver miR-148a to promote proliferation and metastasis of glioblastoma via targeting CADM1. Bull Cancer. 2018;105(7-8):643-51.

59. Saito K, Ohta S, Kawakami Y, Yoshida K, Toda M. Functional analysis of KIF20A, a potential immunotherapeutic target for glioma. J Neurooncol. 2017;132(1):63-74.

60. Tang J, Xu J, Zhi Z, Wang X, Wang Y, Zhou Y, Chen R. MiR-876-3p targets KIF20A to block JAK2/STAT3 pathway in glioma. Am J Transl Res. 2019;11(8):4957-66

61. Heimberger AB, McGary EC, Suki D, Ruiz M, Wang H, Fuller GN, Bar-Eli M. Loss of the AP-2alpha transcription factor is associated with the grade of human gliomas. Clin Cancer Res. 2005;11(1):267-72.

62. Huang W, Zhong Z, Luo C, Xiao Y, Li L, Zhang X, Yang L, Xiao K, Ning Y, Chen $L$, et al. The miR-26a/AP-2alpha/Nanog signaling axis mediates stem cell self-renewal and temozolomide resistance in glioma. Theranostics. 2019;9(19):5497-516.

63. Cao F, Zhang Q, Chen W, Zheng F, Ran Q, He Y, Gao Y, Yao S. miR$6743-5 p$, as a direct upstream regulator of GRIM-19, enhances proliferation and suppresses apoptosis in glioma cells. Biosci Rep. 2017;37:6.

64. Cheng J, Meng J, Zhu L, Peng Y. Exosomal noncoding RNAs in Glioma: biological functions and potential clinical applications. Mol Cancer. 2020;19(1):66

65. Qian M, Wang S, Guo X, Wang J, Zhang Z, Qiu W, Gao X, Chen Z, Xu J, Zhao R, et al. Hypoxic glioma-derived exosomes deliver microRNA-1246 to induce M2 macrophage polarization by targeting TERF2IP via the STAT3 and NF-kappaB pathways. Oncogene. 2020;39(2):428-42.

66. Cancer Genome Atlas Research N: Comprehensive genomic characterization defines human glioblastoma genes and core pathways. Nature 2008, 455(7216): 1061-1068.

67. Huang T, Alvarez AA, Pangeni RP, Horbinski CM, Lu S, Kim SH, James CD, Brenann CW, et al. A regulatory circuit of miR-125b/miR-20b and Wnt signalling controls glioblastoma phenotypes through FZD6-modulated pathways. Nat Commun. 2016;7:12885

68. Ohno M, Natsume A, Kondo Y, Iwamizu H, Motomura K, Toda H, Ito M, Kato T, Wakabayashi T. The modulation of microRNAs by type I IFN through the activation of signal transducers and activators of transcription 3 in human glioma. Mol Cancer Res. 2009;7(12):2022-30.

69. Ray S, Coulter DW, Gray SD, Sughroue JA, Roychoudhury S, Mclntyre EM, Chaturvedi NK, Bhakat KK, Joshi SS, McGuire TR, et al. Suppression of STAT3 NH2 -terminal domain chemosensitizes medulloblastoma cells by activation of protein inhibitor of activated STAT3 via de-repression by microRNA-21. Mol Carcinog. 2018;57(4):536-48.

70. Han L, Yue X, Zhou X, Lan FM, You G, Zhang W, Zhang KL, Zhang CZ, Cheng JQ, Yu SZ, et al. MicroRNA-21 expression is regulated by betacatenin/STAT3 pathway and promotes glioma cell invasion by direct targeting RECK. CNS Neurosci Ther. 2012;18(7):573-83.

71. Wang YY, Sun G, Luo H, Wang XF, Lan FM, Yue X, Fu LS, Pu PY, Kang CS, Liu N, et al. MiR-21 modulates hTERT through a
STAT3-dependent manner on glioblastoma cell growth. CNS Neurosci Ther. 2012;18(9):722-8.

72. Xue J, Zhou A, Wu Y, Morris SA, Lin K, Amin S, Verhaak R, Fuller G, Xie $K$, Heimberger AB, et al. miR-182-5p Induced by STAT3 Activation Promotes Glioma Tumorigenesis. Cancer Res. 2016;76(14):4293-304

73. Yin J, Ge X, Shi Z, Yu C, Lu C, Wei Y, Zeng A, Wang X, Yan W, Zhang J, et al. Extracellular vesicles derived from hypoxic glioma stem-like cells confer temozolomide resistance on glioblastoma by delivering miR-30b-3p. Theranostics. 2021;11(4):1763-79.

74. Mathew LK, Huangyang P, Mucaj V, Lee SS, Skuli N, Eisinger-Mathason TS, Biju K, Li B, Venneti S, Lal P, et al. Feedback circuitry between miR-218 repression and RTK activation in glioblastoma. Sci Signal. 2015:8(375):42.

75. Wang $\mathrm{H}$, Feng $\mathrm{H}$, Zhang $\mathrm{Y}$. Resveratrol inhibits hypoxia-induced glioma cell migration and invasion by the p-STAT3/miR-34a axis. Neoplasma. 2016;63(4):532-9.

76. Zhang $X$, Ding $H$, Han $Y$, Sun D, Wang $H$, Zhai XU. The significance of microRNA-184 on JAK2/STAT3 signaling pathway in the formation mechanism of glioblastoma. Oncol Lett. 2015;10(6):3510-4.

77. Chang JH, Hwang YH, Lee DJ, Kim DH, Park JM, Wu HG, Kim IA. MicroRNA-203 Modulates the Radiation Sensitivity of Human Malignant Glioma Cells. Int J Radiat Oncol Biol Phys. 2016;94(2):412-20.

78. Zhou RJ, Xu XY, Liu BX, Dai WZ, Cai MQ, Bai CF, Zhang XF, Wang LM, Lin L, Jia SZ, et al. Growth-inhibitory and chemosensitizing effects of microRNA-31 in human glioblastoma multiforme cells. Int J Mol Med. 2015;36(4):1159-64.

79. Chen L, Li H, Han L, Zhang K, Wang G, Wang Y, Liu Y, Zheng Y, Jiang T, Pu $P$, et al. Expression and function of miR-27b in human glioma. Oncol Rep. 2011;26(6):1617-21.

80. Yang CM, Chiba T, Brill B, Delis N, von Manstein V, Vafaizadeh V, Oellerich T, Groner B. Expression of the miR-302/367 cluster in glioblastoma cells suppresses tumorigenic gene expression patterns and abolishes transformation related phenotypes. Int J Cancer. 2015;137(10):2296-309.

81. Zheng F, Wang M, Li Y, Huang C, Tao D, Xie F, Zhang H, Sun J, Zhang C, Gu C, et al. CircNR3C1 inhibits proliferation of bladder cancer cells by sponging miR-27a-3p and downregulating cyclin D1 expression. Cancer Lett. 2019;460:139-51.

82. Kopp F, Mendell JT. Functional classification and experimental dissection of long noncoding RNAs. Cell. 2018;172(3):393-407.

83. Zhu J, Fu H, Wu Y, Zheng X. Function of IncRNAs and approaches to IncRNA-protein interactions. Sci China Life Sci. 2013;56(10):876-85.

84. Engreitz JM, Haines JE, Perez EM, Munson G, Chen J, Kane M, McDonel PE, Guttman M, Lander ES. Local regulation of gene expression by IncRNA promoters, transcription and splicing. Nature. 2016;539(7629):452-5

85. Shi J, Dong B, Cao J, Mao Y, Guan W, Peng Y, Wang S. Long non-coding RNA in glioma: signaling pathways. Oncotarget. 2017;8(16):27582-92.

86. Liu H, Li C, Yang J, Sun Y, Zhang S, Yang J, Yang L, Wang Y, Jiao B. Long noncoding RNA CASC9/miR-519d/STAT3 positive feedback loop facilitate the glioma tumourigenesis. J Cell Mol Med. 2018;22(12):6338-44.

87. Zheng J, Liu X, Wang P, Xue Y, Ma J, Qu C, Liu Y. CRNDE promotes malignant progression of glioma by attenuating miR-384/PIWIL4/STAT3 Axis. Mol Ther. 2016;24(7):1199-215.

88. Tang J, Yu B, Li Y, Zhang W, Alvarez AA, Hu B, Cheng SY, Feng H: TGF-beta-activated IncRNA LINC00115 is a critical regulator of glioma stem-like cell tumorigenicity. EMBO Rep 2019, 20(12):e48170.

89. Wu W, Yu T, Wu Y, Tian W, Zhang J, Wang Y. The miR155HG/miR-185/ ANXA2 loop contributes to glioblastoma growth and progression. J Exp Clin Cancer Res. 2019;38(1):133.

90. Liang J, Liu N, Xin H. Knockdown long non-coding RNA PEG10 inhibits proliferation, migration and invasion of glioma cell line $\mathrm{U} 251$ by regulating miR-506. Gen Physiol Biophys. 2019;38(4):295-304.

91. Cao W, Liu B, Ma H. Long non-coding RNA GHET1 promotes viability, migration and invasion of glioma cell line U251 by down-regulation of miR-216a. Eur Rev Med Pharmacol Sci. 2019;23(4):1591-9.

92. Dong N, Guo J, Han S, Bao L, Diao Y, Lin Z. Positive feedback loop of IncRNA HOXC-AS2/miR-876-5p/ZEB1 to regulate EMT in glioma. Onco Targets Ther. 2019;12:7601-9.

93. Hu YW, Kang CM, Zhao JJ, Nie Y, Zheng L, Li HX, Li X, Wang Q, Qiu YR. LnCRNA PLAC2 down-regulates RPL36 expression and blocks cell cycle 
progression in glioma through a mechanism involving STAT1. J Cell Mol Med. 2018;22(1):497-510.

94. Petkovic S, Muller S. RNA circularization strategies in vivo and in vitro. Nucleic Acids Res. 2015;43(4):2454-65.

95. Du WW, Zhang C, Yang W, Yong T, Awan FM, Yang BB. Identifying and Characterizing circRNA-Protein Interaction. Theranostics. 2017;7(17):4183-91

96. Zhou ZB, Huang GX, Fu Q, Han B, Lu JJ, Chen AM, Zhu L. circRNA.33186 contributes to the pathogenesis of osteoarthritis by sponging miR127-5p. Mol Ther. 2019;27(3):531-41.

97. Ashwal-Fluss R, Meyer M, Pamudurti NR, Ivanov A, Bartok O, Hanan M, Evantal N, Memczak S, Rajewsky N, Kadener S. circRNA biogenesis competes with pre-mRNA splicing. Mol Cell. 2014;56(1):55-66.

98. Zheng Q, Bao C, Guo W, Li S, Chen J, Chen B, Luo Y, Lyu D, Li Y, Shi $\mathrm{G}$, et al. Circular RNA profiling reveals an abundant circHIPK3 that regulates cell growth by sponging multiple miRNAs. Nat Commun. 2016;7:11215

99. He Q, Zhao L, Liu X, Zheng J, Liu Y, Liu L, Ma J, Cai H, Li Z, Xue Y. MOV10 binding circ-DICER1 regulates the angiogenesis of glioma via miR103a-3p/miR-382-5p mediated ZIC4 expression change. J Exp Clin Cancer Res. 2019;38(1):9

100. Lei B, Huang Y, Zhou Z, Zhao Y, Thapa AJ, Li W, Cai W, Deng Y. Circular RNA hsa_circ_0076248 promotes oncogenesis of glioma by sponging miR-181a to modulate SIRT1 expression. J Cell Biochem. 2019;120(4):6698-708.

101. Wang Y, Sui X, Zhao H, Cong L, Li Y, Xin T, Guo M, Hao W. Decreased circular RNA hsa_circ_0001649 predicts unfavorable prognosis in glioma and exerts oncogenic properties in vitro and in vivo. Gene. 2018;676:117-22.

102. Hu D, Zhang Y. Circular RNA HIPK3 promotes glioma progression by binding to miR-124-3p. Gene. 2019;690:81-9.
103. Wang J, Zhang Y, Song H, Yin H, Jiang T, Xu Y, Liu L, Wang H, Gao H, Wang $R$, et al. The circular RNA circSPARC enhances the migration and proliferation of colorectal cancer by regulating the JAK/STAT pathway. Mol Cancer. 2021;20(1):81

104. Zhang X, Yang J, Bian Z, Shi D, Cao Z. Long noncoding RNA DANCR promotes nasopharyngeal carcinoma progression by interacting with STAT3, enhancing IL-6/JAK1/STAT3 signaling. Biomed Pharmacother. 2019;113:108713.

105. Tan Y, Wang Y, Zou Y, Zhou C, Yi Y, Ling Y, Liao F, Jiang Y, Peng X. LncRNA LOC101927514 regulates PM25-driven inflammation in human bronchial epithelial cells through binding p-STAT3 protein. Toxicol Lett. 2020:319:119-28.

106. Liu J, Yang Q, Sun H, Wang X, Saiyin H, Zhang H. The circ-AMOTL1/ENO1 axis implicated in the tumorigenesis of OLP-associated oral squamous cell carcinoma. Cancer Manag Res. 2020;12:7219-30.

107. Tang WG, Hu B, Sun HX, Sun QM, Sun C, Fu PY, Yang ZF, Zhang X, Zhou $\mathrm{CH}$, Fan J, et al. Long non-coding RNA00364 represses hepatocellular carcinoma cell proliferation via modulating p-STAT3-IFIT2 signaling axis. Oncotarget. 2017;8(60):102006-19.

108. Guo X, Xue H, Shao Q, Wang J, Guo X, Chen X, Zhang J, Xu S, Li T, Zhang $P$, et al. Hypoxia promotes glioma-associated macrophage infiltration via periostin and subsequent $\mathrm{M} 2$ polarization by upregulating TGF-beta and M-CSFR. Oncotarget. 2016;7(49):80521-42.

109. Zhang Y, Li X, Zhang J, Liang H. Natural killer T cell cytotoxic activity in cervical cancer is facilitated by the LINC00240/microRNA-124-3p/ STAT3/MICA axis. Cancer Lett. 2020;474:63-73.

\section{Publisher's Note}

Springer Nature remains neutral with regard to jurisdictional claims in published maps and institutional affiliations.
Ready to submit your research? Choose BMC and benefit from:

- fast, convenient online submission

- thorough peer review by experienced researchers in your field

- rapid publication on acceptance

- support for research data, including large and complex data types

- gold Open Access which fosters wider collaboration and increased citations

- maximum visibility for your research: over $100 \mathrm{M}$ website views per year

At BMC, research is always in progress.

Learn more biomedcentral.com/submissions 